\title{
Monitoring moored instrument motion by optimal estimation
}

\author{
Fabienne Gaillard $^{\mathrm{a}, *}$, Thierry Terre ${ }^{\mathrm{a}}$, Antoine Guillot ${ }^{\mathrm{b}}$ \\ a Laboratoire de physique des océans, BP 70, 29280 Plouzané, France \\ ${ }^{\mathrm{b}}$ Division technique de l'INSU, BP 74, 29280 Plouzané, France
}

Received 29 January 2004; accepted 25 April 2005

Available online 14 July 2005

\begin{abstract}
Many oceanographic applications require the positioning of the underwater sensor at measurement times. We consider here the case of subsurface moored tomographic instruments, where the distance between source and receiver must be known within a few meters. For that purpose, a long baseline array is deployed: this system includes a navigator, attached to the mooring element and an array of three transponders set on the ocean bottom. To process the navigation data collected with such system, we have developed a method based on optimal estimation. The triangulation problem is not a basic spherical constraints one and the specificity of deep underwater positioning, related to the variability of the ocean sound speed profile are pointed out. Correcting terms are proposed and introduced into the system. Simultaneous inversion of all data, defining an overconstrained problem allows to estimate biases and errors. The algorithm is applied here to a dataset collected in the Azores-Canary basin during CAMBIOS experiment.

(C) 2005 Elsevier Ltd. All rights reserved.
\end{abstract}

Keywords: Underwater acoustic positioning; Acoustic transponder survey; Long baseline array

\section{Introduction}

The problem of monitoring underwater displacements is part of many applications such as deployment of underwater vehicles or free falling instruments, but it also appears in

\footnotetext{
* Corresponding author.

E-mail addresses: fabienne.gaillard@ifremer.fr (F. Gaillard), thierry.terre@ifremer.fr (T. Terre), antoine. guillot@ifremer.fr (A. Guillot).
}

0029-8018/\$ - see front matter (C) 2005 Elsevier Ltd. All rights reserved. 
the case of anchored devices. The mooring lines set to hold instruments such as current meters, temperature sensors or tomographic sources or receivers, lean under the strong currents or move with the tides or inertial effects. We present here a method developed in the context of physical oceanography. It has been applied to the CAMBIOS experiment that took place in the Canary basin from july 1997 to april 1998, as part of european project CANIGO. In order to measure integral heat content and detect temperature anomalies, acoustic tomography devices separated by hundreds of kilometers were deployed for approximately 9 months. The accurate positioning of the tomographic instruments, although not absolutely necessary, makes the data processing easier and improves the quality of the analysis by removing one unknown from the system. To be of real use, the accuracy of the positioning has to be of the order of a few meters. For that purpose, the tomography intruments included a navigation system and a long base line array was deployed on the seabed below each mooring. The calibration of the base line array, which means determining the absolute position of each bottom transponder was performed at the beginning of the experiment. During the 9 months of the experiment, the navigation system sent acoustic pulses to the array of bottom transponders and recorded the corresponding round trip travel times.

Many aspects of the calibration problem have been explored by Send et al. (1995). The present work is a generalization of the method called 'absolute inversion method' in their paper. Here, both aspects of the positioning problem: inital calibration of the base line array and tracking of the instrument over the 9 month duration of the experiment, are considered in a unified framework and the particular processing of each dataset will share the same methodology. Moreover, the ocean variability has been taken into account. We aimed at setting up a practical method, offering the tradeoff between easy use, with relatively light computational cost, and satisfying the accuracy requirements. The paper presents the basic relations, or model, relating the measurements to the parameters which are to be estimated, then we introduce the most currently used approximations, and check their validity. We propose a correcting term when it appears to be necessary. The positioning problem is solved by an optimal estimation method. The travel time dataset is combined with independent data when available, and a priori knowledge is introduced in the covariance matrices.

A first version of the algorithm was developped at LPO by Guillot (1992) for processing the THETIS- 1 data collected in the Mediterranean. A number of new features have been added while analyzing the CAMBIOS dataset which is presented here and the method is now implemented in the TOMOLAB sofware (Octopus, 2001).

\section{The problem of acoustic positioning}

\subsection{Relating travel times to coordinates}

In the general configuration, the transducer of the navigation system onboard a vehicle either at surface (ship), or at an intermediate depth (underwater mooring) transmits an acoustic signal to an array of bottom transponders. Each transponder answers and the round trip travel time is recorded. Assuming that all instrument delays are known, 
and the geometry has not changed, the one-way travel time from the top transponder to the bottom transponder can be extracted. In order to localize the different elements in space, one needs to relate those travel times to horizontal and vertical distances with a formula as simple as possible, so as to be inverted with no or little iterations.

Acoustic paths in the ocean undergo refraction due to variations of sound speed with depth that can be accurately reproduced by ray-tracing. In the problem considered here the horizontal and vertical ranges are similar and the angles with respect to vertical are of the order of $\pi / 4$ or smaller and only direct paths are involved. For these large angles, refraction is moderate and can be approximated to avoid systematic use of a ray-tracing code.

Travel time and ray path computations depend on the sound speed profile, that may vary greatly between different areas of the world ocean. Since we present here the navigation data collected on CAMBIOS mooring T1 (Fig. 1), the sound speed profile obtained at this point will be used to illustrate the processing. Hydrographic measurements (temperature, pressure and salinity) have been performed in the vicinity of mooring T1. The corresponding vertical profile of sound speed can be deduced from those variables by applying one of the recommended formulae for the speed of sound. Dushaw et al. (1993) have evaluated the validity of these formulae. They concluded that Del Grosso formula is accurate within $0.05 \mathrm{~m} / \mathrm{s}$, over the deep ocean pressure ranges while Chen-Millero formula overestimates the speed of sound by $0.4 \mathrm{~m} / \mathrm{s}$ at depth greater than $4000 \mathrm{~m}$. We selected Del Grosso to compute the sound speed profile at T1, presented in Fig. 2. To illustrate the profiles variability, several profiles taken during the same cruise and data from Reynaud

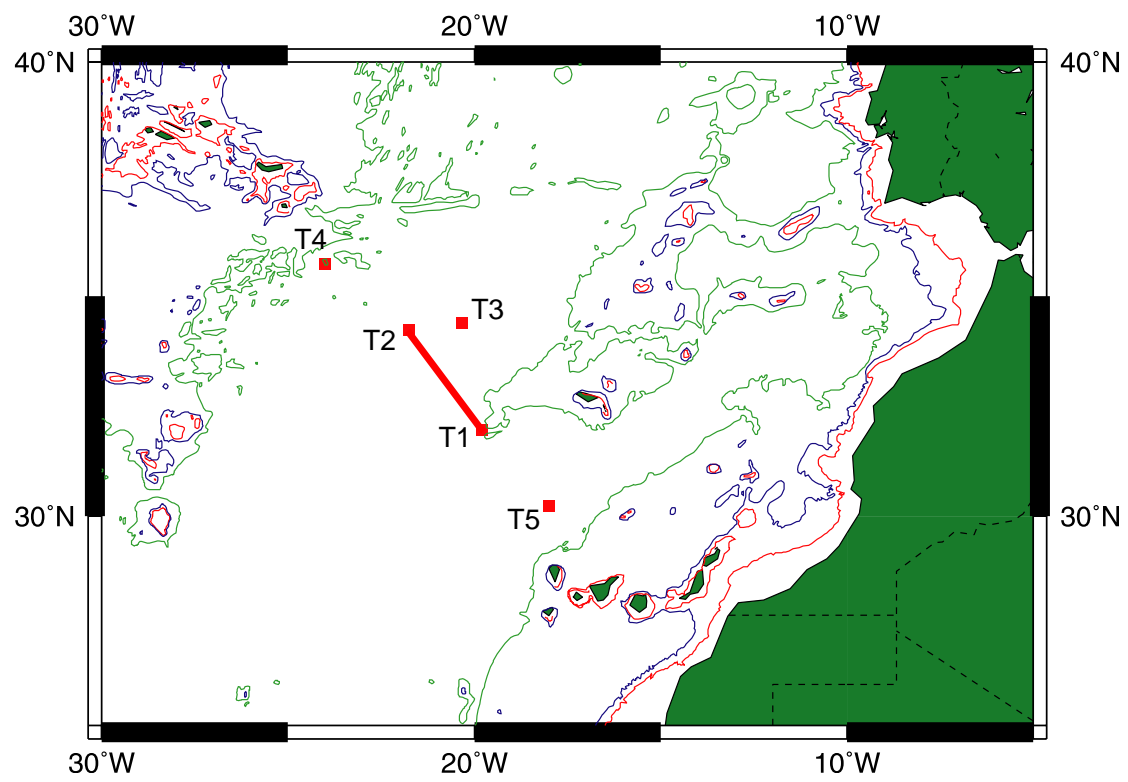

Fig. 1. Geographical area of CAMBIOS experiment and position of the moorings. The processing of navigation data collected at point $\mathrm{T} 1$ is presented here. 


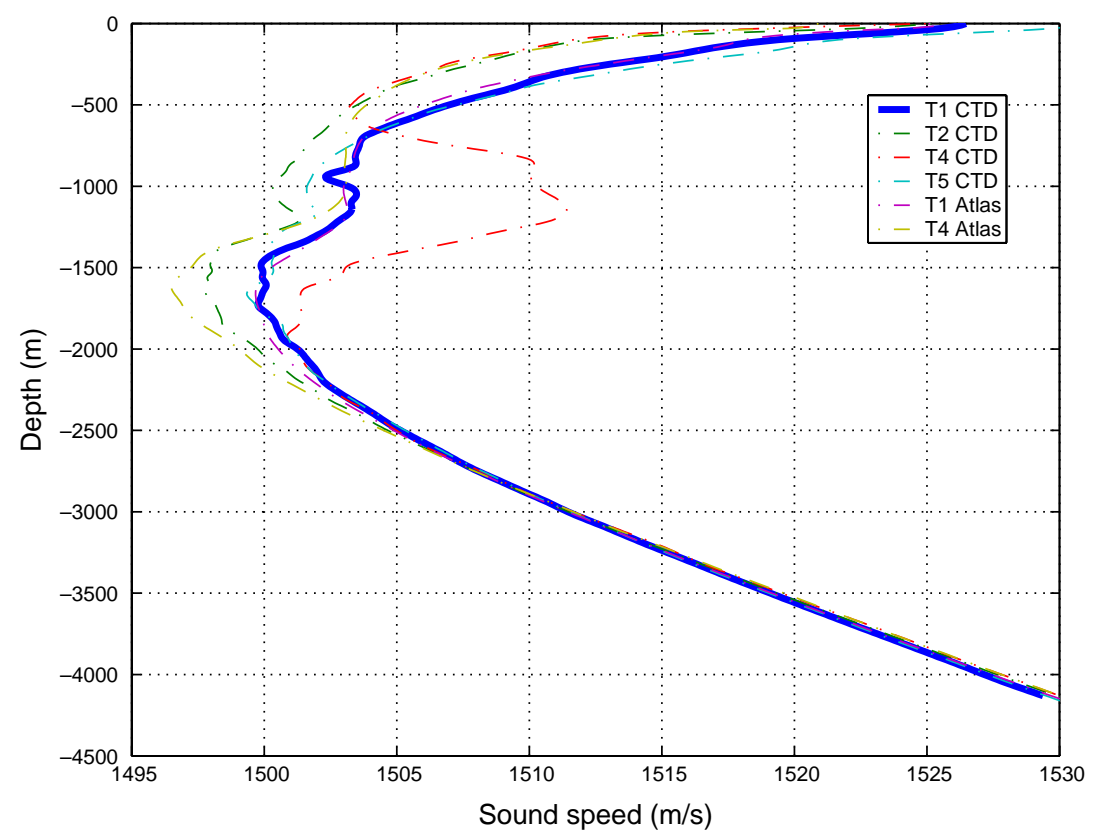

Fig. 2. Sound speed profiles deduced from temperature and salinity measurement. The heavy line is the profile measured at $\mathrm{T} 1$ before the calibration survey. Other profiles were measured during CAMBIOS cruise at different moorings (T2, T4, T5). The profiles extracted from Reynaud atlas at points T1 and T4 are also shown. T4 profile exhibits a strong anomaly near $1000 \mathrm{~m}$, it is produced by an eddy of Mediterranean water (meddy).

atlas (Reynaud et al., 1998) are overlaid. In this area, the sound speed may vary by several $\mathrm{m} / \mathrm{s}$ over the upper $2000 \mathrm{~m}$.

The acoustic travel time $\tau$ from the top transponder, located at depth $z_{t}$, to the bottom transponder, located at depth $z_{b}$, is given by an integral computed along the ray path. Introducing $\theta$, the ray angle with respect to the horizontal, gives:

$$
\tau=\int_{z_{t}}^{z_{b}} \frac{\mathrm{d} z}{C(z) \sin (\theta)}
$$

On flat earth, the simplest computation could be done by assuming straight path between top and bottom transponders, this path is called slant range, noted $L$, it is related to the depth $D$ and horizontal distance on the earth surface $H$ by: $L^{2}=H^{2}+D^{2}$. The first approximation for travel time uses a mean sound speed $\left(C_{0}\right)$, in such case the travel time is:

$$
\tau_{0}=L C_{0}^{-1}
$$

The ray curvature due to refraction can be approximated by introducing the harmonic sound speed $C_{h}$ :

$$
C_{h}=\left(\frac{1}{z_{b}-z_{t}} \int_{z_{t}}^{z_{b}} \frac{\mathrm{d} z}{C(z)}\right)^{-1}
$$


The travel time becomes:

$$
\tau_{1}=L C_{h}^{-1}
$$

On a spherical earth, the horizontal distance is shorter at depth than it is on the earth surface (or sea level), for that reason, an earth curvature correction is introduced in the ray tracing codes written in cartesian coordinates (Aki and Richards, 1980), by stretching the sound speed profile. Similarly, the earth curvature can be taken into account in the straight path approximation by correcting the slant range $L$. Introducing the earth radius $R_{\mathrm{e}}$ at the top transponder depth, and $\phi$, the azimuthal angle, gives:

$$
L^{2}=2 R_{\mathrm{e}}\left(D-R_{\mathrm{e}}\right)(\cos (\phi)-1)+D^{2}
$$

which can be approximated by:

$$
L^{2}=H^{2}\left(1-D / R_{\mathrm{e}}\right)+D^{2}
$$

leading to the travel time formula:

$$
\tau_{2}=\left[H^{2}\left(1-D / R_{\mathrm{e}}\right)+D^{2}\right]^{1 / 2} C_{h}^{-1}
$$

The range of validity of the various approximations is explored for the sound speed profile observed at T1, assuming the source at the ocean surface and the receiver at $4000 \mathrm{~m}$ depth and for horizontal distances up to $10 \mathrm{~km}$. The travel times obtained by ray tracing, and considered as the reference, are compared with the three approximations of increasing realism: (1) the straight line approximation using a mean sound speed: $\tau_{0}$, (2) the harmonic approximation $\tau_{1}$, (3) the harmonic approximation with the earth curvature correcting term $\tau_{2}$. The results are shown in Fig. 3. The harmonic sound speed alone corrects the travel time up to $1 \mathrm{~km}$ range, then the error starts to grow. If we take the criterion of allowing less than half a millisecond error, it appears that the earth curvature correction is needed when the horizontal range reaches $4 \mathrm{~km}$ and holds up to $10 \mathrm{~km}$. We will use $\tau_{2}$ approximation in the subsequent computations.

The relation between the acoustic travel time $\tau$ and the coordinates of the transducer $\left(x_{t}, y_{t}, z_{t}\right)$ and the bottom transponder $\left(x_{b}, y_{b}, z_{b}\right)$ is approximated by:

$$
\sqrt{\left(\left(x_{b}-x_{t}\right)^{2}+\left(y_{b}-y_{t}\right)^{2}\right)\left(1-\frac{z_{b}-z_{t}}{R_{\mathrm{e}}}\right)+\left(z_{b}-z_{t}\right)^{2}}=(\tau+\varepsilon) * C_{h} .
$$

The right hand side of the equation contains the observation and the associated error $\varepsilon$ that includes measurement errors and modeling approximations. The left hand side contains the unknown coordinates.

\subsection{Solving the non-linear problem}

We gather the system unknowns (instrument coordinates) in a single vector, to define the state vector $\mathbf{x}$, and the data (travel times) to define the observation vector $\mathbf{y}^{\circ}$, to express the previous relation in the unified notation described by Ide et al. (1997). The system can 

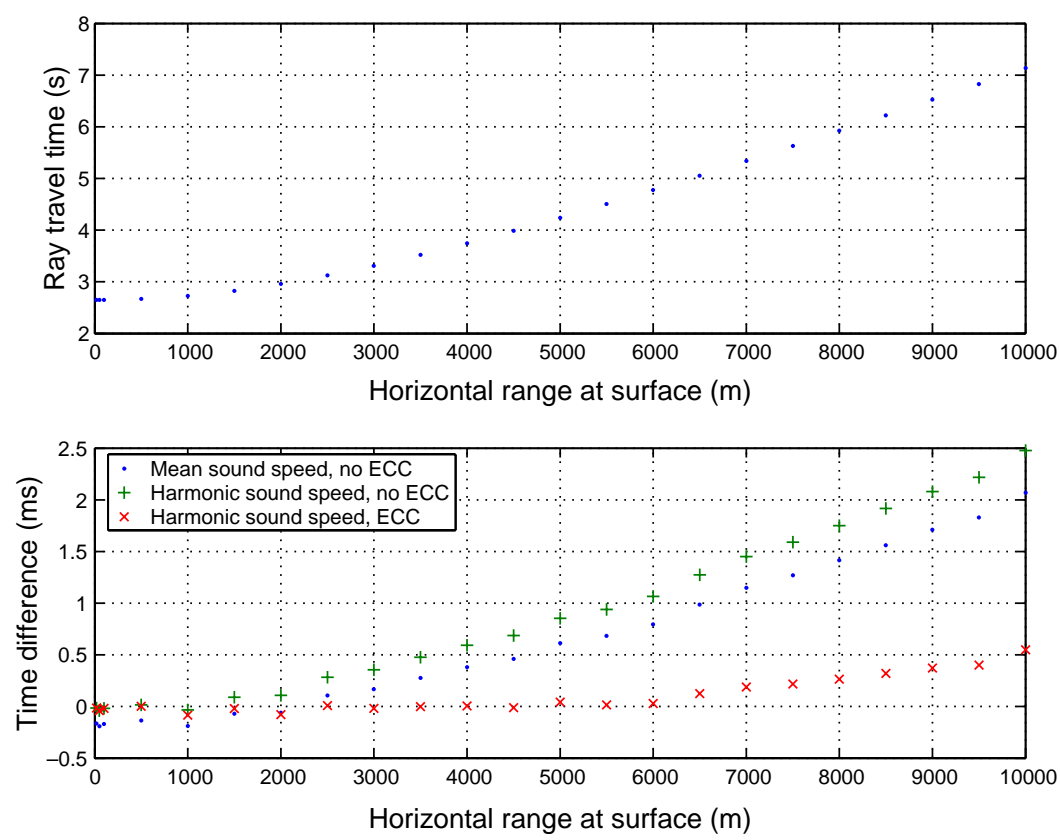

Fig. 3. Top panel: Travel times computed by a ray tracing program taking into account earth curvature. This travel time is used as the reference. Bottom panel: travel time differences with the reference travel time in the case of different approximations. Straight line approximation with mean sound speed, $\tau_{0}$ (dots), harmonic approximation, $\tau_{1}(+)$, harmonic approximation with earth curvature correcting term, $\tau_{2}(\mathrm{X})$.

be represented by a relation of the form:

$$
H(\mathbf{x})-\mathbf{y}^{\circ}=\varepsilon .
$$

Under the assumption of gaussian distribution of all variables and errors, the maximum likelihood solution is obtained by minimizing the cost function:

$$
J(\mathbf{x})=\left(\mathbf{y}^{\circ}-H(\mathbf{x})\right)^{t} \mathbf{R}^{-1}\left(\mathbf{y}^{\circ}-H(\mathbf{x})\right)+\left(x-x_{b}\right)^{t} \mathbf{B}^{-1}\left(\mathbf{x}-\mathbf{x}_{b}\right)
$$

where $\mathbf{x}_{b}$ is some a priori (or background) estimate of $\mathbf{x}, \mathbf{B}$ the matrix of a priori covariances of $\mathbf{x}$ and $\mathbf{R}$ the covariance matrix of the measurement errors.

If the curvature correcting term $\left(z_{b}-z_{t}\right) / R_{\mathrm{e}}$ is replaced by its background value, the function $H$ is quadratic and the problem can be solved iteratively by linearizing around a previous solution $\mathbf{x}_{n}$ (Tarantola and Valette, 1982):

$$
H\left(\mathbf{x}_{n}+\mathrm{d} X\right)=H\left(\mathbf{x}_{n}\right)+\mathbf{H}_{n} \mathrm{~d} X+O\left(|\mathrm{~d} X|^{2}\right),
$$

where $\mathbf{H}_{n}$ is the matrix of partial derivatives of $H$ at point $\mathbf{x}_{n}$. The minimum of $J(\mathbf{x})$ provides the solution, with the corresponding a posteriori error:

$$
\mathbf{x}_{n+1}=\mathbf{x}_{b}+\mathbf{K}_{n}\left(\mathbf{y}^{\circ}-H\left(\mathbf{x}_{n}\right)+\mathbf{H}_{n}\left(\mathbf{x}_{n}-\mathbf{x}_{b}\right)\right), \quad \mathbf{P}_{n+1}=\left(I-\mathbf{K}_{n} \mathbf{H}_{n}\right) \mathbf{B}
$$


where

$$
\mathbf{K}_{n}=\mathbf{B H}_{n}^{t}\left(\mathbf{R}+\mathbf{H}_{n} \mathbf{B} \mathbf{H}_{n}^{t}\right)^{-1}
$$

The iteration is stopped when the new point $\mathbf{x}_{n+1}$ is within a prescribed distance of the previous point $\mathbf{x}_{n}$.

\section{Calibrating the long baseline array}

A ship survey has been performed during the first CAMBIOS cruise to determine the position of the three transponders deployed on the ocean bottom. The calibration system included a command/range unit, also used for mooring releases, and PCs for collecting data from acoustic interrogations and GPS ship navigation. A transducer installed under the ship hull was sending acoustic pings every $15 \mathrm{~s}$ while the ship was underway. Fig. 4 shows the ship track during the survey. It is to notice that this track results from a crude tradeoff between the various constraints imposed by the cruise within the limited ship time. A study of the observation matrix $\mathbf{H}$ would certainly lead to propose a more efficient path, this point has not been considered here. The round trip travel times from the ship transducer to the bottom transponders have been acquired and stored together with GPS ship positions and headings. The measured round trip travel times to each transponder $\tau_{i}^{r m}$

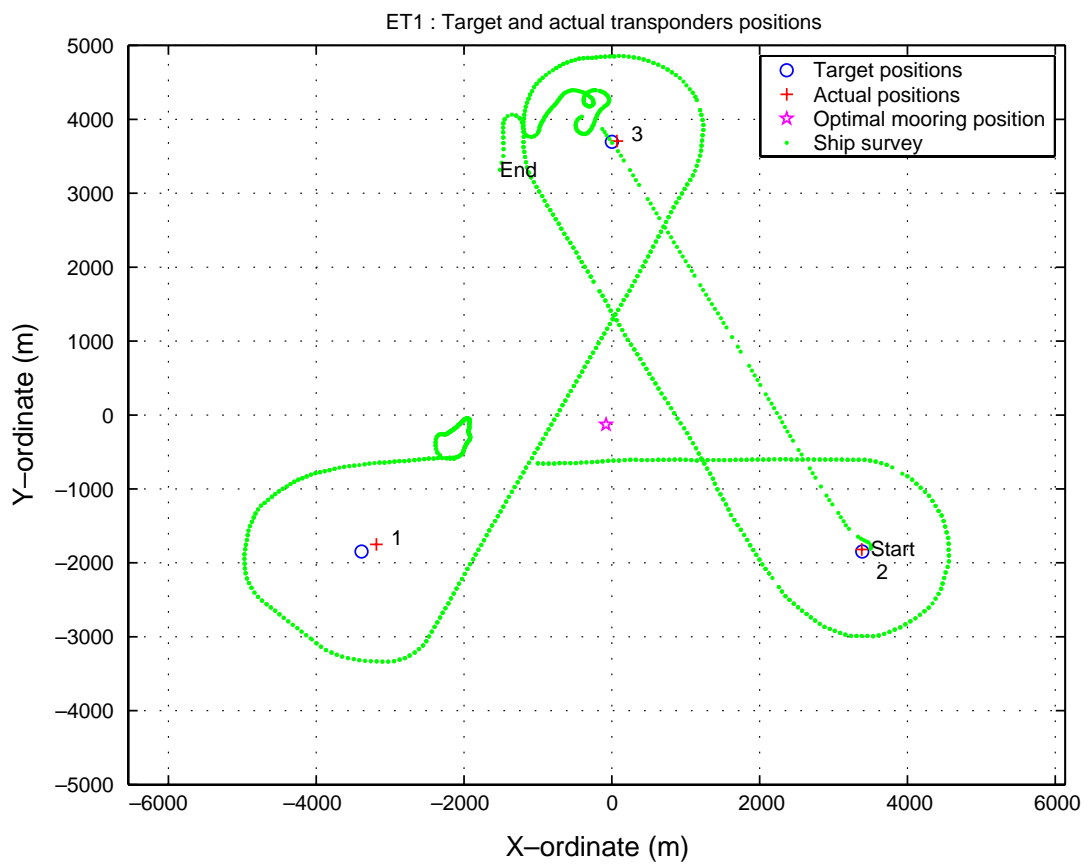

Fig. 4. Ship track during transponders survey. Round trip travel time from all operating transponders has been recorded at each position, while the ship was underway. 


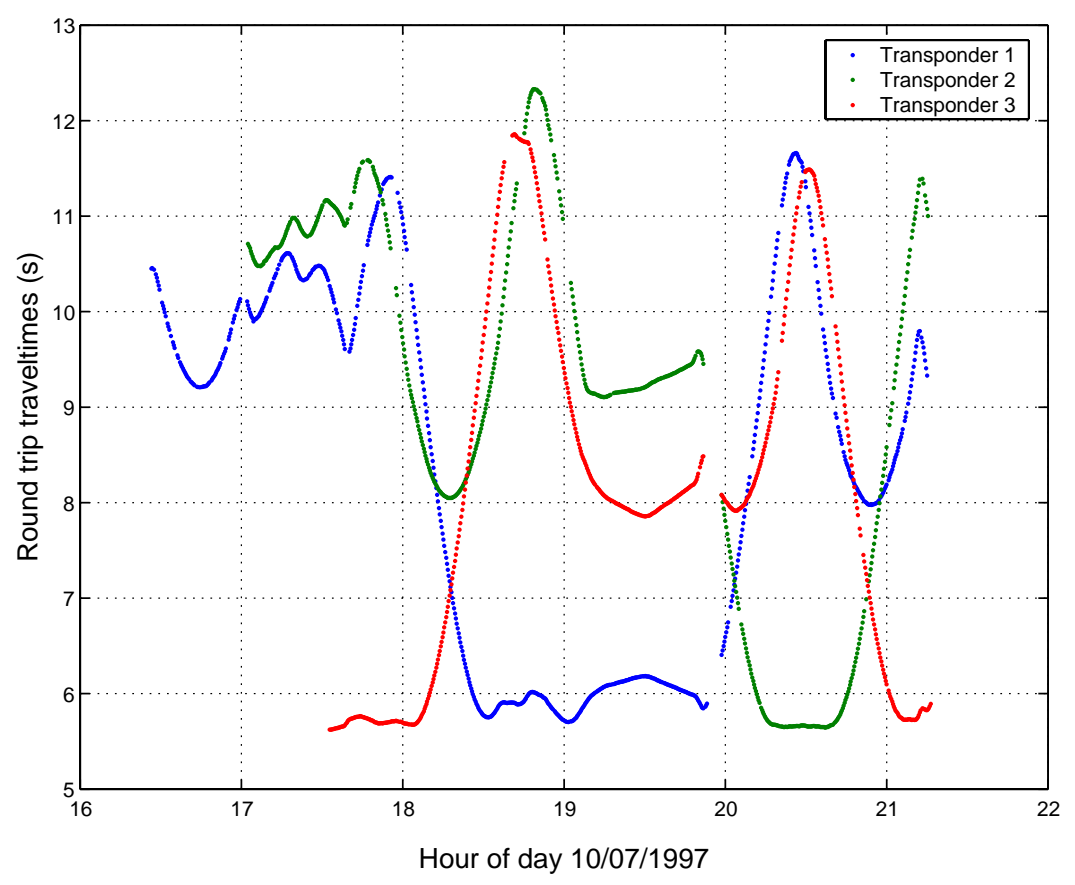

Fig. 5. Round trip travel times collected at each interrogation time.

are shown in Fig. 5. To obtain the one-way travel time between the transducer and the bottom transponder, several corrections must be applied.

(1) The transducer position at transmission time is obtained by correcting the ship GPS position from the offset between the GPS antenna and the acoustic transducer, using the ship heading data.

(2) The instrumental delays $(d)$ on travel times introduced by the system are removed.

$$
\tau_{i}^{r}=\tau_{i}^{r m}-d
$$

(3) The round trip travel times are measured while the ship is steaming at a speed ranging from 5 to 10 knots (Fig. 6). The ping is transmitted when the ship is at the recorded GPS position, when the bottom transponder reply arrives the ship has traveled a distance that may reach $60 \mathrm{~m}$. Consequently the round trip travel time is:

$$
\tau_{i}^{r}=\tau_{i}+\tau_{i}^{\prime}
$$

where $\tau_{i}$ is the one-way travel time from the transducer position to the bottom transponder, and $\tau_{i}^{\prime}$ the return travel time, from the bottom transponder to the transducer new (and unknown) position. A first method for correcting this effect would be to interpolate the ship position and heading at the correct intermediate time between transmission and reception. We chose to correct the travel times by using 


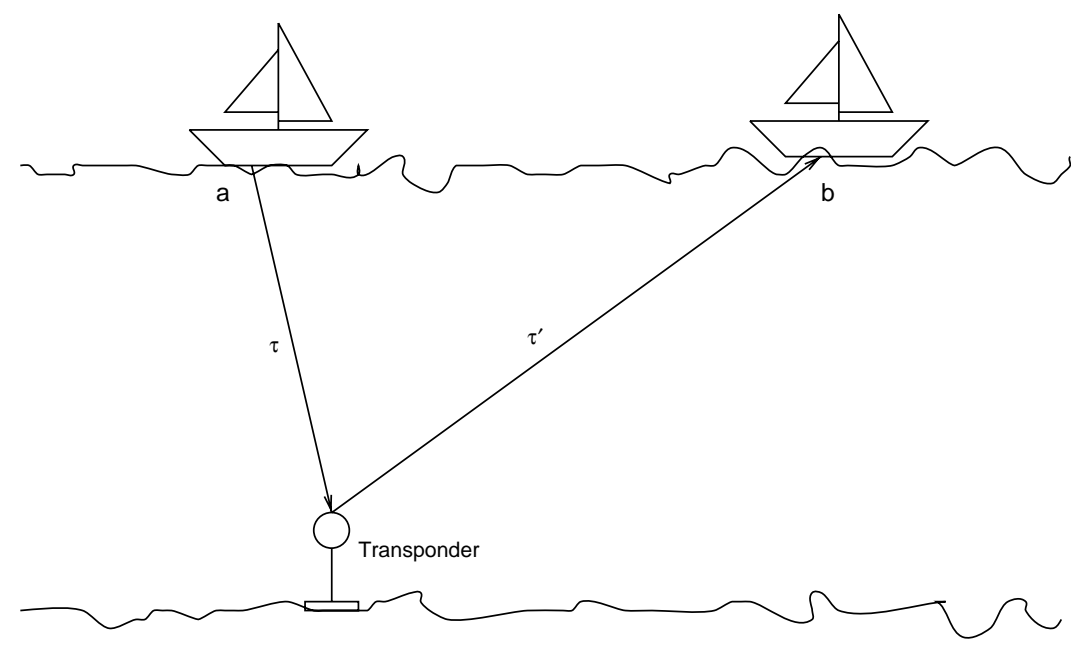

Fig. 6. Effect of ship displacement on travel time measurements: geometry of the problem. The ship GPS position is recorded when the ping is transmitted (a) but the answer from the transponder arrives when the ship is in (b).

the rate of change of the measured round trip travel times during the survey, to estimate the one-way travel time $\tau_{i}$ associated with the GPS position.

$$
\tau_{i}^{\prime}=\tau_{i}+\tau_{i}^{r} \frac{\mathrm{d} \tau_{i}^{r}}{\mathrm{~d} t}
$$

then the one-way travel time is given by:

$$
\tau_{i}=\frac{\tau_{i}^{r}}{2}\left(1-\frac{\mathrm{d} \tau_{i}^{r}}{\mathrm{~d} t}\right)
$$

The effect of the correcting terms: GPS antenna, earth curvature and ship displacement, are shown in Fig. 7, where they have been converted to range. The dominant correction is the effect of ship displacement. The earth curvature term, although small is important because its neglect could introduce a bias that would not be averaged out by the method.

For each interrogation, we collected up to three answers. Each travel time is related to the instrument coordinates by an equation of the form:

$$
\sqrt{\left(\left(x_{j}-x_{i}^{s}\right)^{2}+\left(y_{j}-y_{i}^{s}\right)^{2}\right)\left(1-\frac{z_{j}-z_{i}^{s}}{R_{\mathrm{e}}}\right)+\left(z_{j}-z_{i}^{s}\right)^{2}}=C_{h j}\left(\tau_{i j}+\varepsilon_{i j}\right) .
$$

where subscript $i$ indicates the ping number, and exponent $s$ a ship coordinate, subscript $j$ the bottom transponder number. In final we obtain a set of 500 equations for estimating three sets of $(\mathrm{x}, \mathrm{y}, \mathrm{z})$ transponder coordinates. One harmonic sound speed $C_{h j}$ is computed 

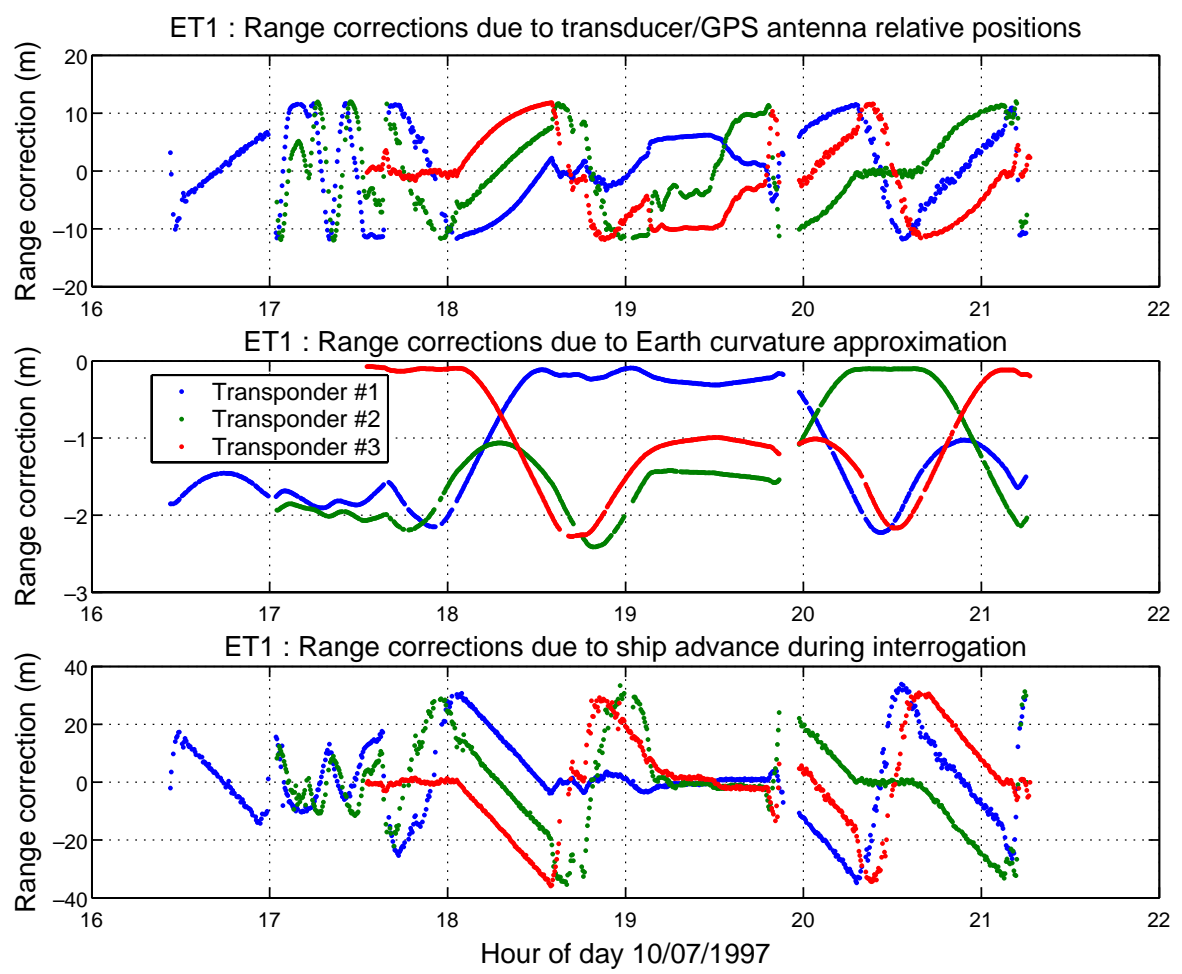

Fig. 7. Corrections modifying the travel time during calibration of the long baseline array. Travel times have been converted to ranges. Corrections due to transducer offset relative to GPS antenna position (top), earth curvature corrections in slant range computations (middle), corrections applied on travel times to compensate for ship displacement converted to range (bottom).

for each transponder depth from the sound speed profile measured during the survey and presented in Fig. 2.

The calibrated position of the transponders is obtained, initializing relation 13 from the target position and iterating. The initial and final coordinates are given Table 1. Only the results corresponding to the corrected travel times are shown. Taking the corrections into account introduces a stretching of the array, each transponder moving 5-10 m away from the center of the array. The compatibility of the model with the data is examined through the residuals displayed in Fig. 8. With uncorrected data, large residuals remain, their structure is correlated with the ship displacement. When all corrections are applied, the RMS value of the residuals corresponds to a range inconsistency of $5 \mathrm{~m}$, in agreement with our a priori estimation of the various errors due to measurements and approximations. Despite the strong reduction in amplitude, the structure of the residuals remains unchanged, indicating a systematic error, likely due to the ship positioning. The positions collected during the experiment were provided by the ship navigation system, based on Differential GPS. A first source of error was that the ground station used for differential GPS was very far (Santa Maria/Azores), but the dominant effect, which certainly explains 
Table 1

Coordinates of the transponder array at various stages of the estimation

\begin{tabular}{|c|c|c|c|}
\hline & B1 & B2 & B3 \\
\hline \multicolumn{4}{|l|}{ Target position } \\
\hline Target latitude & N 3159.000 & N 3159.000 & N 3202.000 \\
\hline Target longitude & W 1950.150 & W 1945.850 & W 1948.000 \\
\hline Target depth & 4150 & 4115 & 4130 \\
\hline \multicolumn{4}{|l|}{ Calibration correction } \\
\hline $\mathrm{X}$ correction $(\mathrm{m})$ & 200 & -4 & 69 \\
\hline Y correction (m) & 96 & 28 & 9 \\
\hline $\mathrm{Z}$ correction $(\mathrm{m})$ & 5 & -19 & -3 \\
\hline \multicolumn{4}{|l|}{ Calibrated position } \\
\hline Calibrated latitude & N 3159.052 & N 3159.015 & N 3202.005 \\
\hline Calibrated longitude & W 1950.023 & W 1945.852 & W 1947.956 \\
\hline Calibrated depth & 4155 & 4096 & 4127 \\
\hline \multicolumn{4}{|l|}{ Navigation correction } \\
\hline dX from nav (m) & -12 & +17 & -5 \\
\hline dY from nav (m) & -8 & -17 & +25 \\
\hline dZ from nav (m) & 0 & 0 & 0 \\
\hline
\end{tabular}
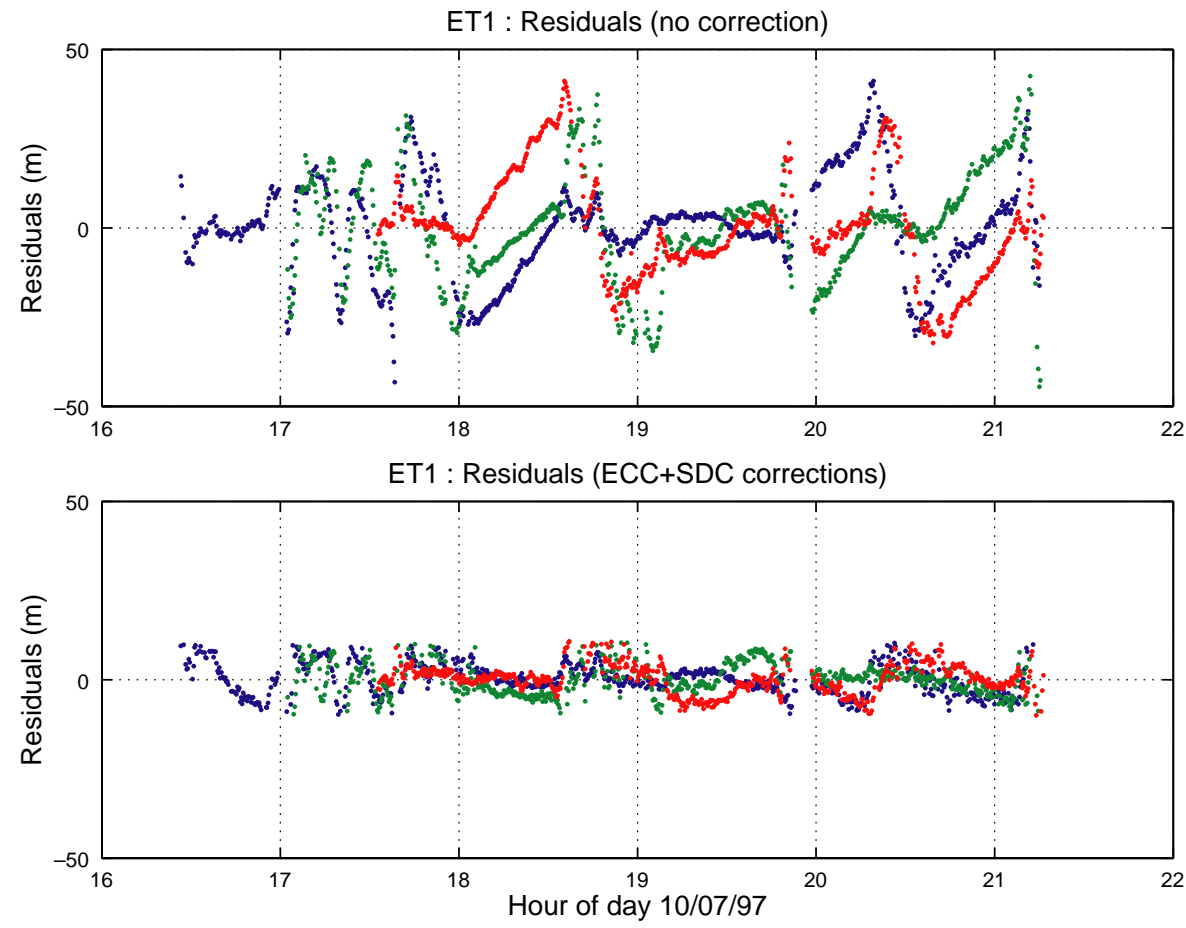

Fig. 8. Residuals on the estimation of the three transponders positions problem computed (top) without corrections and (bottom) with Earth Curvature and Ship Displacement Corrections (SDC). 
the strong relation with the ship displacement, is the integration time of the GPS system of the ship, that did not allow for an accurate instantaneous positioning of a moving object.

\section{Monitoring mooring motions}

We will now focus on the reconstruction of the trajectory of the tomographic instrument set on mooring line T1. Four time series have been recorded by the instrument: three series of round trip travel times between the navigator and the three bottom transponders plus a series of pressure. They are shown in Fig. 9. At first glance, we identify three time scales in the signal: two of them are periodic and linked to tides and inertial oscillations, the third scale is related to mesoscale events.

Knowing the position of this fixed bottom transponder array, we can follow the trajectory of the instrument over the duration of the experiment. In that case, the unknowns of the system are the three navigators coordinates at each time of interrogation:

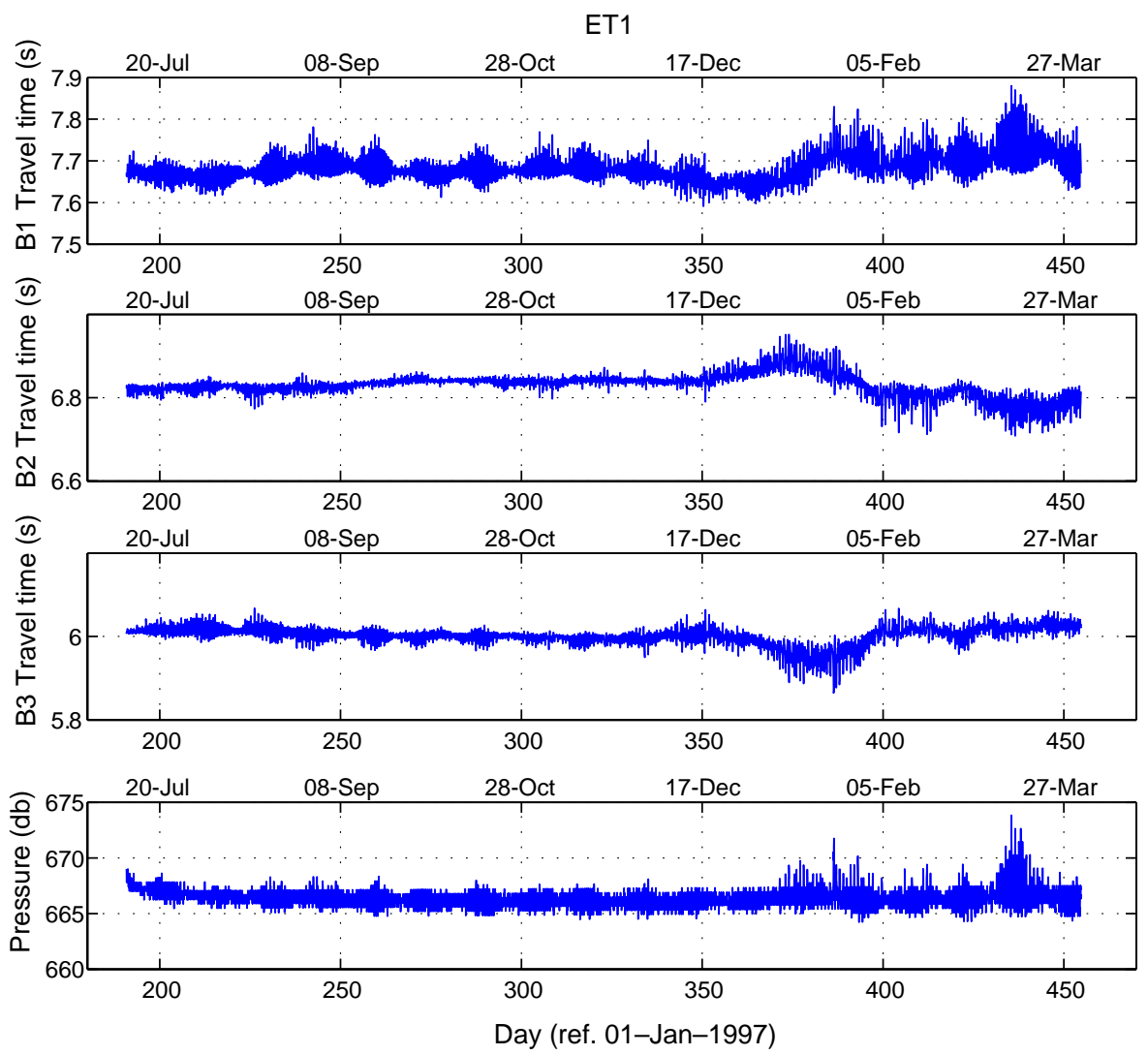

Fig. 9. Data available for navigating the instrument: Data series of round trip travel times to bottom transponders recorded by the navigator set on T1 (first 3 panels). Pressure recorded in T1 tomography instrument (bottom). 
$\left(x_{m i}, y_{m i}, z_{m i}\right)$. If the positions of the bottom transponders are not perfectly known, their coordinates $\left(x_{j}, y_{j}, z_{j}\right)$ are added to the vector of unknowns. The data are the travel times $\tau_{i j}$, with corresponding errors noted $\varepsilon_{i j}$. The equation between navigation data and unknown coordinates is:

$$
\sqrt{\left(\left(x_{m i}-x_{j}\right)^{2}+\left(y_{m i}-y_{j}\right)^{2}\right)\left(1-\frac{z_{j}-z_{m i}}{R_{e}}\right)+\left(z_{m i}-z_{j}\right)^{2}}=C_{h i j}\left(\tau_{i j}+\varepsilon_{i j}\right)
$$

At each interrogation, three round trip travel times are collected, and the full vector of unknowns is:

$$
\mathbf{x}_{i}=\left[x_{m i}, y_{m i}, z_{m i}, x_{1}, y_{1}, z_{1}, x_{2}, y_{2}, z_{2}, x_{3}, y_{3}, z_{3}\right]^{T} ;
$$

The depth of the navigator was followed by a pressure sensor (Fig. 9), this data is added to the system by converting pressure to depth (knowing the water density), providing a fourth equation:

$$
z_{m i .}=z_{p i}+\varepsilon_{p i}
$$

The solution given by Eq. (13), must be initialized with a first guess. For the first navigation time, the first guess for the instrument position is given by the mooring nominal position estimated during the mooring deployment, the depth is deduced from the bathymetry and wire length. At subsequent times, we use the estimate of the position obtained at the previous acoustic interrogation. The covariance matrices are built by combining the a posteriori error on the previous position and a probable displacement with an amplitude determined by the time lag between two interrogations and an estimated maximum mooring velocity.

\subsection{Transponder position assumed correct}

A first estimate is done by assuming that the calibration survey has provided exact transponder positions. We use the same harmonic sound speed as for the calibration, over the whole experiment. The residuals produced by the estimation do not exhibit the expected behavior of having zero mean, being uncorrelated and randomly distributed, but show instead a clear bias (Fig. 10). This behavior reveals a discrepancy, constant over the duration of the experiment, between the model and the data. The most probable explanation to this bias is attributed to an error in the position of the bottom transponders.

In addition to this constant offset, a strong increase in the residuals occurs between days 350 and 400. Such time dependency resembles the ocean variability, and can be related to a change in temperature and/or salinity. Measurements obtained from temperature and salinity recorders set at various depth between 500 and $3500 \mathrm{~m}$ on the mooring line confirmed that a strong signal in temperature $\left(+1.5^{\circ}\right)$ occurred at depths of $1000 \mathrm{~m}$ and $1200 \mathrm{~m}$ during that period (Gaillard et al. 1999). This corresponds to an intrusion of Mediterranean water in the area which produces a local maximum of sound speed at that level. The magnitude of residuals observed during this warm event is compatible with the effect of sound speed variability on travel time. It clearly appears that the sound speed profile cannot be assumed constant over the 9 months of the experiment and that we need 
ET1 : Daily residuals on travel times (optimal [0 0 0 $]$ )
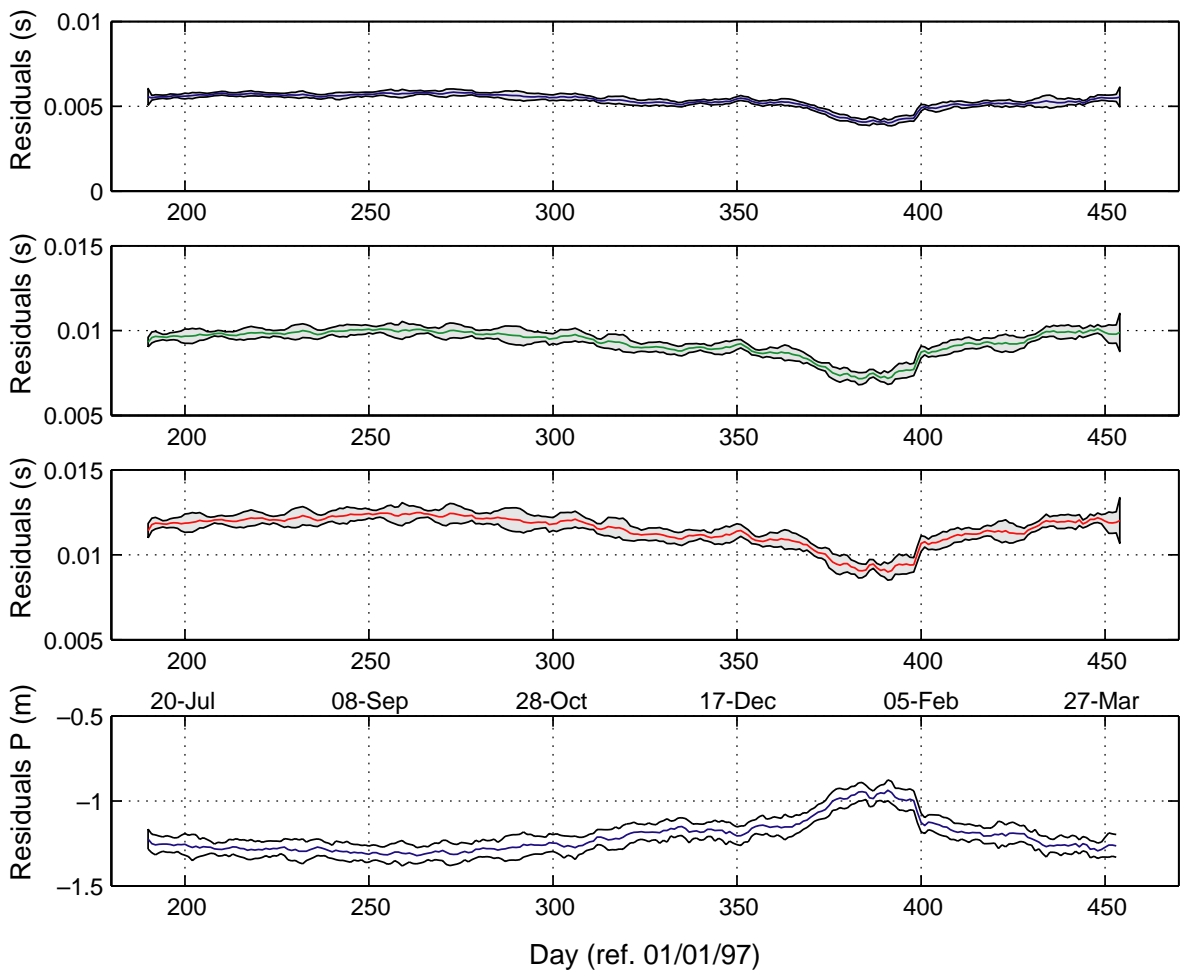

Fig. 10. Residuals on data obtained from the navigation done assuming calibration of the long baseline array was exact (daily averages and corresponding RMS).

to introduce some information about the variability. The temperature series obtained by the instruments set on the mooring line provide the necessary information. Combining the profile measured at the initial time, with the time series of anomalies with respect to this profile, we obtain the time series of harmonic sound speed for each transponder shown in Fig. 11.

\subsection{Improving transponder position}

A second run of the algorithm is performed to optimize the position of the bottom transponders. For doing so, they are allowed to move slightly by introducing a slow velocity. The varying harmonic sound speeds are used in that case. Starting from the 'calibrated position', the transponders converge slowly toward a new position located $\sim 20 \mathrm{~m}$ apart (see Fig. 12). Since the transponders are in fact at a fixed position, we estimate that this final position is an improved position deduced from a survey done by the mooring itself. We have to admit that the positions deduced from the calibration survey do not agree with the navigation data. The navigation estimate can be biased by a constant offset. We excluded a pressure sensor offset, since it has been calibrated before and after 


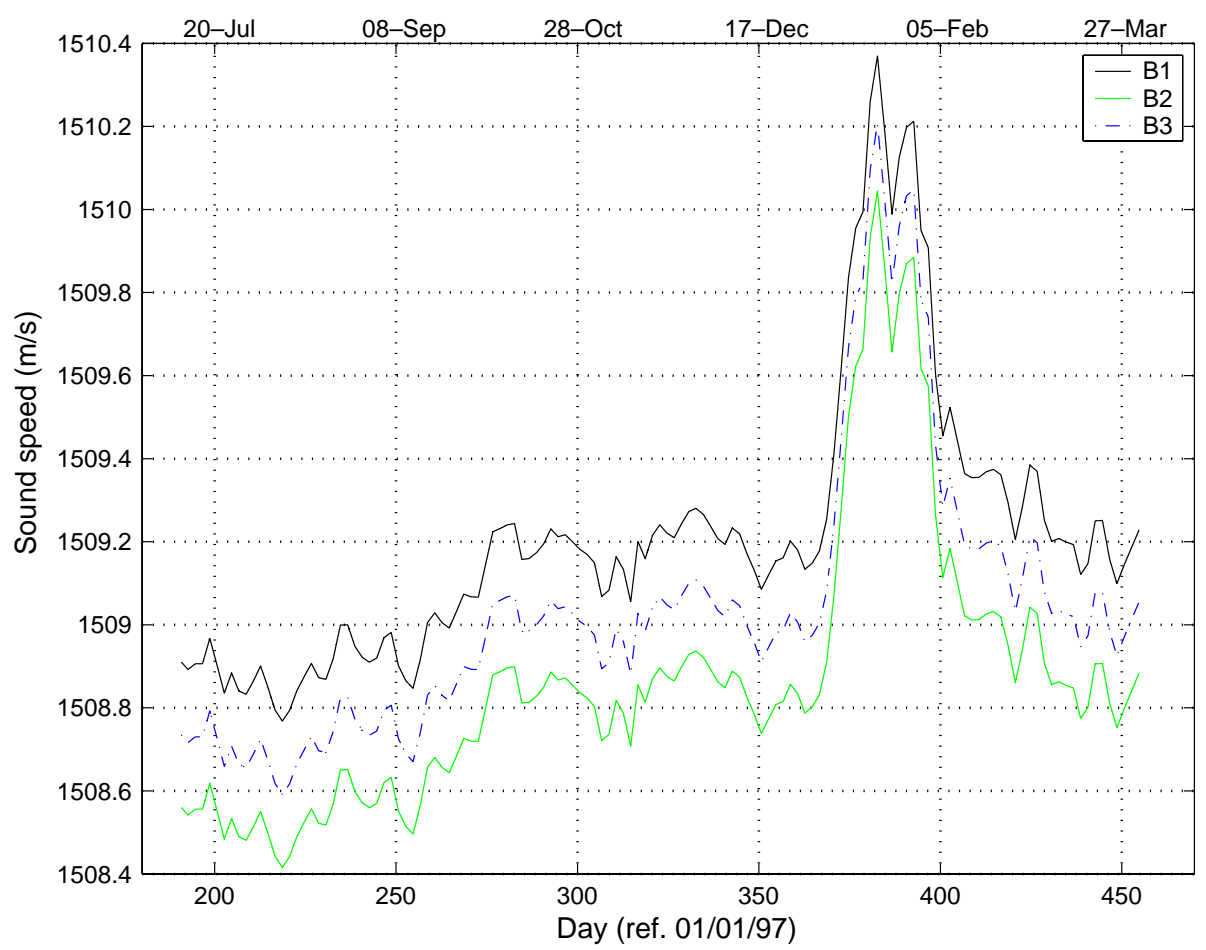

Fig. 11. Time varying harmonic sound speed for each transponders computed from the intial sound speed profile and the temperature variability measured by the sensors deployed on the mooring line.

the experiment, and the measurement agrees with the estimate of the mooring length and bottom depth. Some internal delay in the navigation system may also be incorrect. If so, a change in the bottom transponders position is an acceptable way of compensating this offset, as long as the exact position of the transponders is not our concern. On the side of the calibration, we had already pointed out possible biases left in the system. The optimized positions will be used for the final estimate of the mooring trajectory (Table 1).

\subsection{Final estimate for the mooring trajectory}

The mooring trajectory obtained while the transponders are allowed to move cannot be taken as a valid solution, especially at the beginning of the time series. A final run, with transponders fixed at their optimized position is performed to obtain the three dimensional trajectory of the tomography instrument over the duration of the experiment. The residuals of the mooring motion obtained in that configuration are shown in Fig. 13. Most of the bias has been removed, the residuals are centered around zero, only a few events of less than a millisecond amplitude remain. The time series of the mooring coordinates (Fig. 14), and the mooring trajectory (Fig. 15) over the whole experiment show that the mooring evolves within a $250 \mathrm{~m}$ side square, describing approximate 


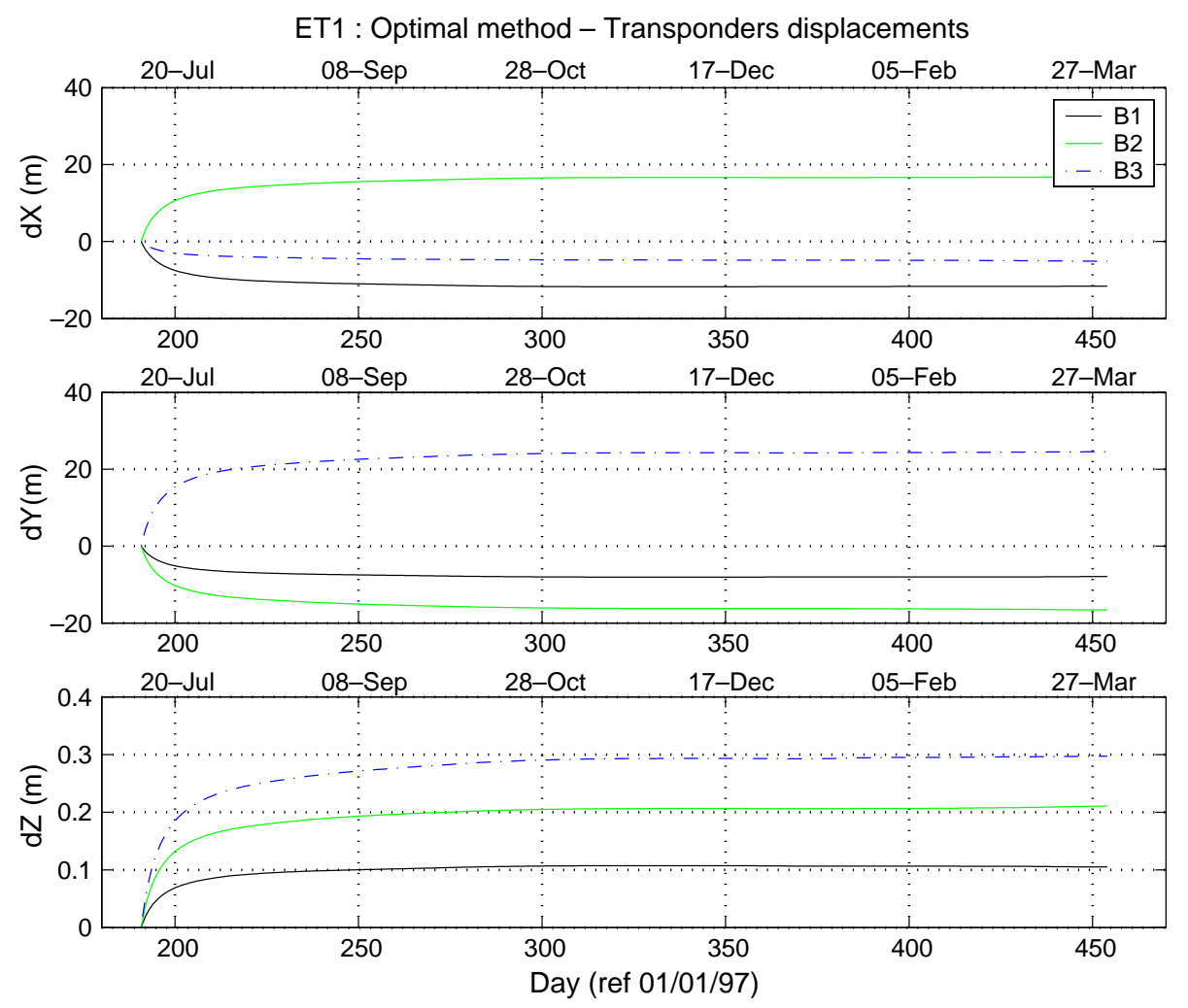

Fig. 12. Improving the transponders position: modification of the coordinates provided by the calibration.

ellipses at the inertial frequency ( $22 \mathrm{~h} \mathrm{40).} \mathrm{The} \mathrm{parameter} \mathrm{of} \mathrm{the} \mathrm{ellipses} \mathrm{(axis} \mathrm{length} \mathrm{and}$ orientation) vary along the experiment, most frequent events correspond to eastward displacements. These time series of coordinates are an interesting tool for studying the behavior of underwater moorings, but the original goal of this processing is to correct the tomography travel times. The motion of mooring $\mathrm{T} 1$ produces a change in the distance between the tomography instruments of pair T1-T2. The travel time of the tomography signal increases or decreases accordingly. Correcting the tomography signal from these changes in distance improves the signal processing: once removed the high frequency variation due to mooring motion, consecutive arrival sequences of tomography are better aligned (or more coherent) and can be averaged to increase signal to noise ratio. The correction is also important to ensure that most of the travel time variations observed in the tomography signal are produced by changes in the ocean sound speed structure. However, we must be aware that a constant bias in the absolute position of the instrument may remain, and has to be taken into account in the tomography data analysis to be carried on. These corrections are obtained by recomputing the distance at all times for a tomographic pair. In the case of pair $\mathrm{T} 1-\mathrm{T} 2$, the time series of travel time correction due only to T1 motion is shown in Fig. 16. 

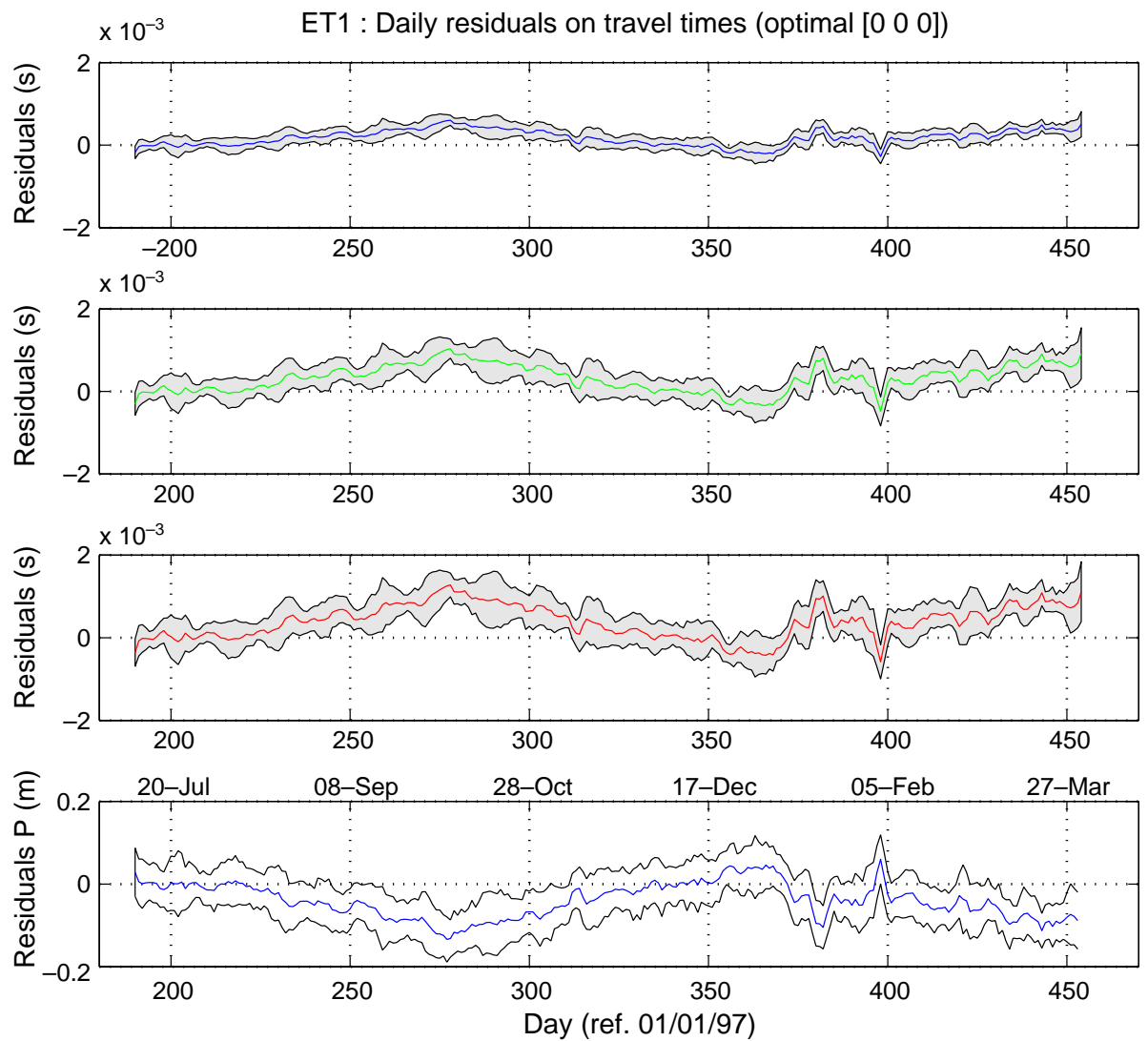

Fig. 13. Residuals on data obtained from the navigation done using a varying harmonic sound speed and optimized transponders position (daily averages and corresponding RMS).

\subsection{Testing sensitivity}

In order to quantify the bias that may remain in our optimal solution, we have analyzed the dependency of the trajectory on the different estimations performed. Fig. 15 shows a one day trajectory, selected in the case of strong displacement, as produced in different localizations: (1) a simple triangulation, based only on three travel times, (2) the first estimation with constant harmonic sound speed and transponders at the position given by the calibration, (3) the final position, considered as optimal. We see that taking into account pressure, or changing transponder position mostly produces a translation of the trajectory.

To evaluate the performance of the algorithm in dealing with the uncertainty in the bottom transponder position, a simple sensitivity test is performed. One transponder (B1) is moved $20 \mathrm{~m}$ eastward from its optimal position. The navigation software is then applied in two cases. In case (a) all transponder positions are assumed unknown, In case (b) only 
ET1 : mooring motions (optimal [0 00 0 $]$ )
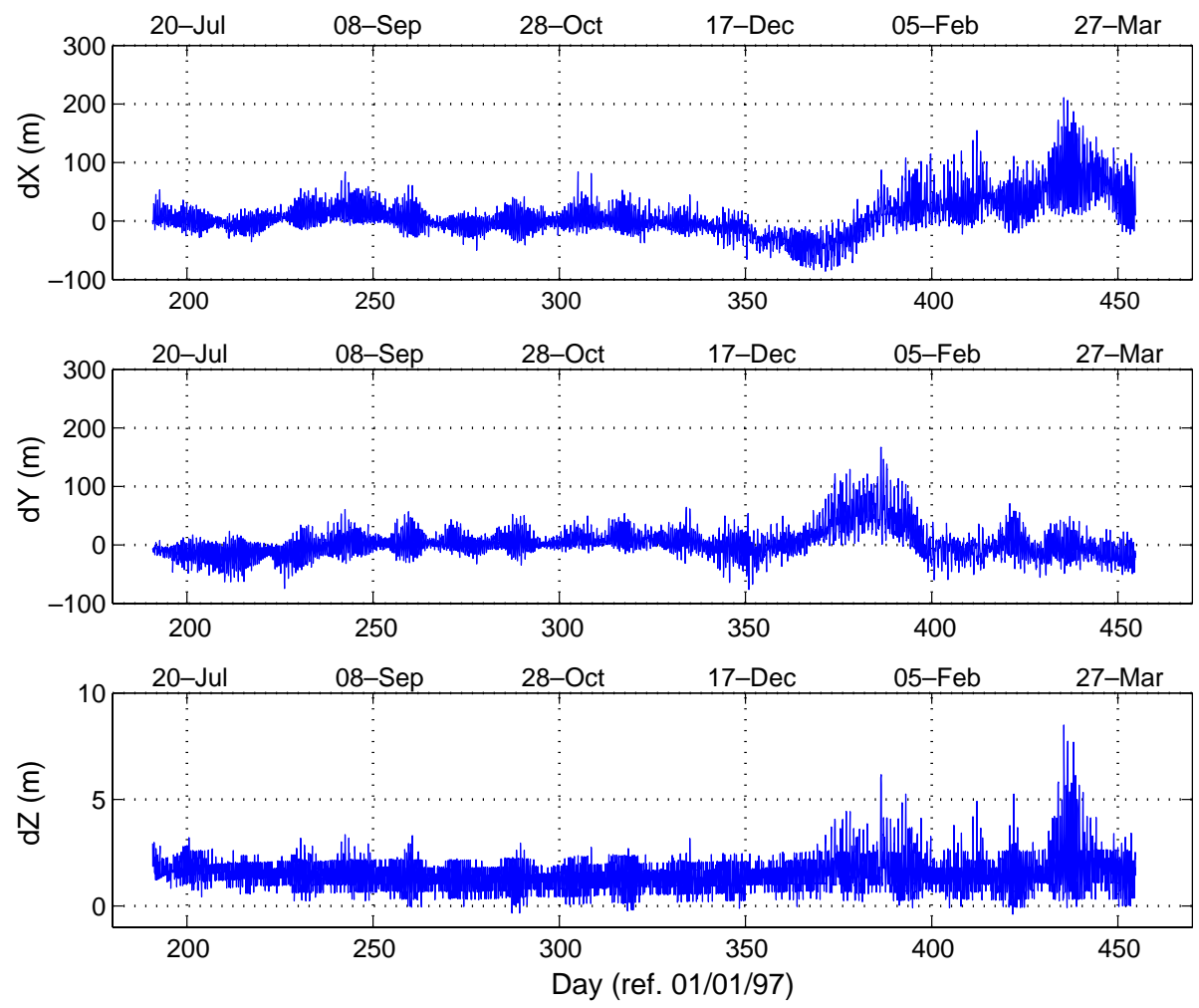

Fig. 14. Mooring motions estimation (X(top), Y(middle), Z(bottom)) around the mooring most probable position.

the position of transponder B1 is estimated. The correction to the initial coordinates of the three transponders are shown in Fig. 17. When the position of all transponders is estimated (case a), the error on the transponder position is dispatched over the three transponders resulting in a change of the center of gravity of the array. If only transponder 1 position is estimated (case b), then the amplitude of the displacement is correct, but the new position is in the East-south-East direction instead of East. The effects of the incorrect tranponder position on the mooring displacements in both cases is visualized in Fig. 15. In case (a) the mooring trajectory is translated by $14 \mathrm{~m}$, in case (b) the trajectory is hardly distinguished from the optimal one, with differences not exceeding $0.7 \mathrm{~m}$.

\section{Conclusion}

The accurate (within a meter) underwater positioning over the full ocean depth is a more complex problem than simple triangulation with circular constraints. The vertical 

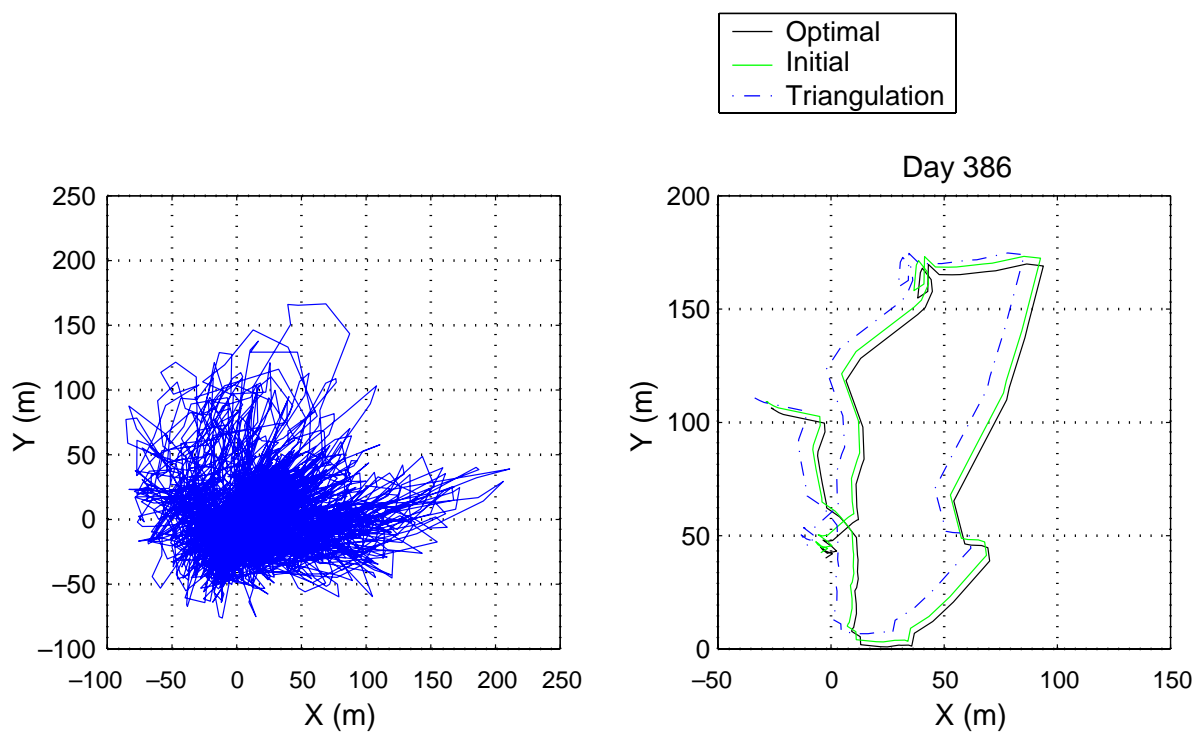

Fig. 15. Left: position of the mooring relative to its most probable position. Right: trajectory during one particular day (plain line: best estimate).

stratification of the ocean induces curvature of the ray path that must be taken into account through the use of harmonic sound speed. Solving the problem in Cartesian coordinates requires correction for the earth curvature. The variation of sound speed with time must also be accounted for. In addition to the complexity of the model that relates the unknown to the data, the measurements, either of acoustic travel times or of GPS position, involve noise and biases. In order to take into account these correcting terms and improve the model by explicitly estimating the biases, we have developed an algorithm that relies on

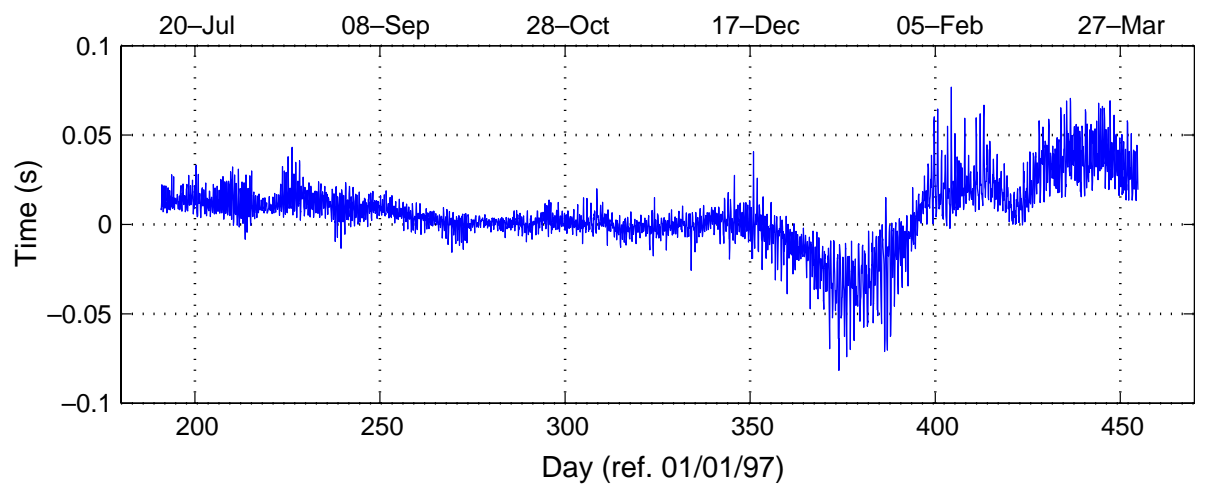

Fig. 16. Travel time correction to pair T1-T2 data due to $\mathrm{T} 1$ motion along the experiment. 

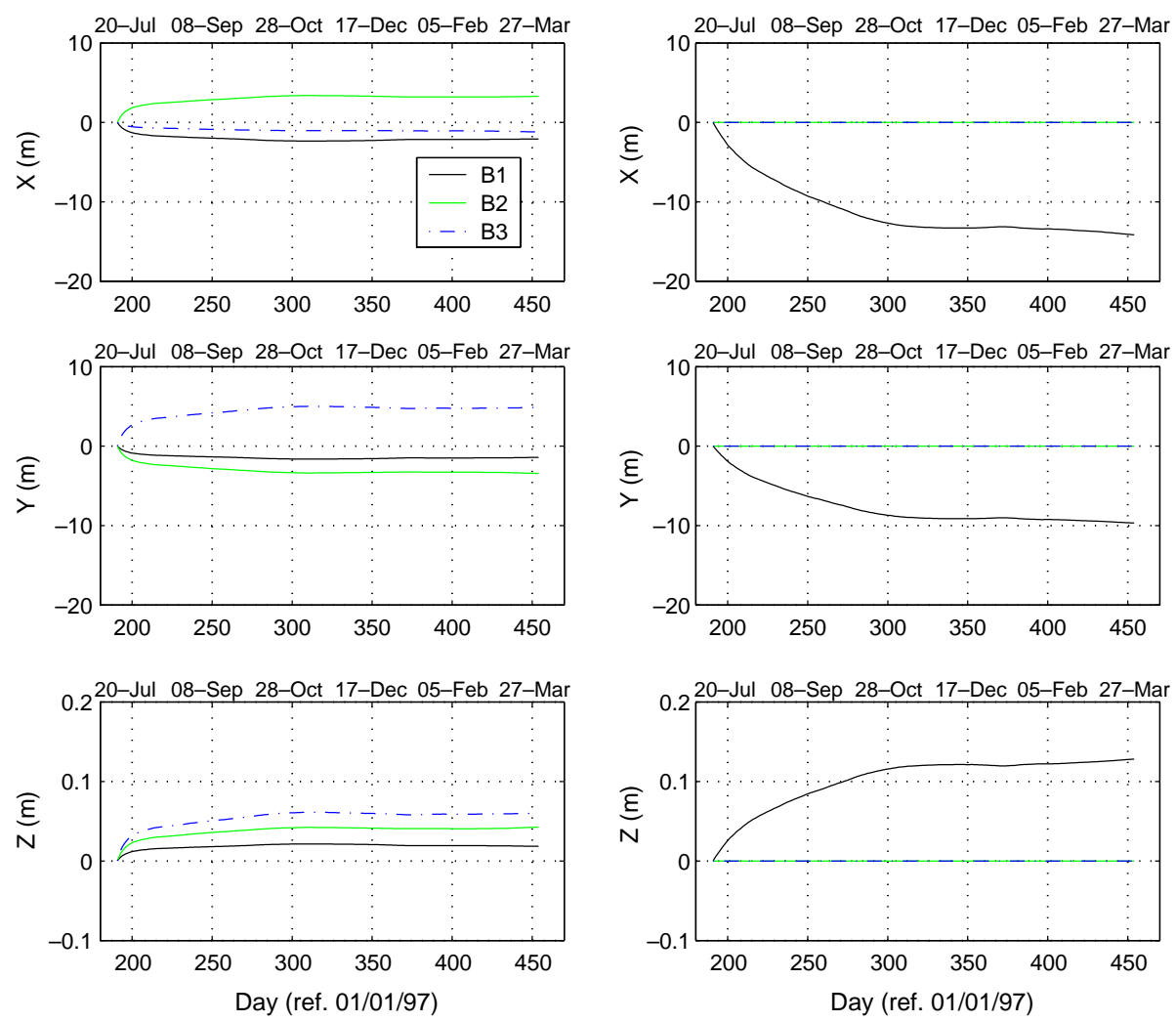

Fig. 17. Correction to the optimized coordinates of the three transponders in the sensitivity test where B1 has been moved 20 m eastward $(d X=20, d Y=d Z=0)$. Left: result when the three transponder position are estimated. Right: result when only transponder 1 position is estimated.

the principles of optimal estimation and takes into account simultaneously all sources of data, Fig. 18.

The method has been applied on real data, collected during CAMBIOS cruise, in the Azores-Canary basin. Both problems of calibrating the transponders array, and positioning the moored instrument have been explored. Starting from the simplest model, the compatibility of the solution with the data and model has been tested by examining the residuals. Discrepancies have appeared in both types of problems. They have been solved by using a more complex model: correcting terms for earth curvature, time varying harmonic sound speed, or by defining new unknowns such as the position of the bottom transponders. The final trajectories produced during the last iteration of the process are recovered with a random error of about one meter. A possible constant bias of the order of 5-10 m remains on the absolute position. The data and the associated information on error and biases will be used to correct the tomographic travel times of the experiment. 


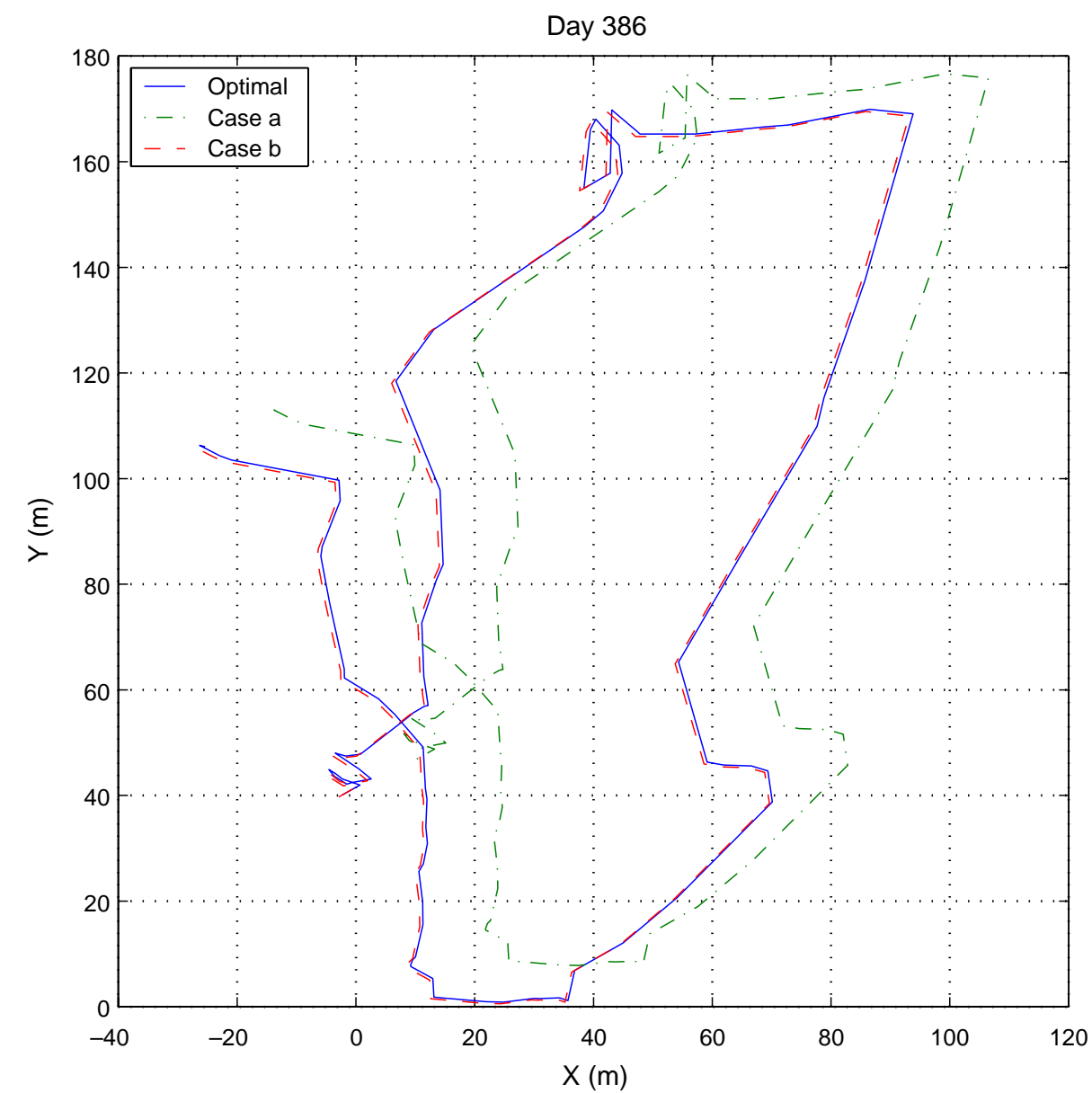

Fig. 18. Mooring trajectory on day 386. Optimal case (plain line) and sensitivity cases. When only B1 position is estimated, the trajectory cannot be distinguished from the optimal.

\section{Acknowledgements}

This work was supported by european MAST III projects CANIGO (MAS3-CT960060) and OCTOPUS (MAS3-CT97-0417).

\section{References}

Aki, K., Richards, P., 1980. Quantitative seismology, theory and methods, vol. 2. Freeman, San Francisco p. 932. Dushaw, B., Worcester, P., Cornuelle, B., Howe, B., 1993. On the equation for the speed of sound in seawater. J. Acoust. Soc. Am. 93 (1), 255-275.

Gaillard, F., Billant, A., Branellec, P. 1999: CAMBIOS contribution à CANIGO (MAS3-CT96-0060) Volume 2: Expérience CAMBIOS, rapport de données eulériennes sur les 4 mouillages, Rapport interne LPO 99-02. 
Guillot, A. 1992: Localisation d'un Mouillage par Méthode de Triangulation, Rapport de Stage DEA Océanographie Physique, Université de Bretagne Occidentale.

Ide, K., Courtier, P., Ghil, M., Lorenc, A.C., 1997. Unified notation for data assimilation: operationnal, sequential and variational. J. Meteorological Soc. Jpn. 75 (1B), 181-189.

Octopus group, 2001: Tomolab, a software package for the design and analysis of ocean acoustic tomography experiments. User's guide.

Reynaud, T., Legrand, P., Mercier, H., Barnier, B. 1998: A new analysis of hydrographic data and its application to an inverse modelling study. Woce Newsletter, Sept 1998.

Send, U., Visbeck, M., Krahman, G., 1995. Aspects of acoustic transponders surveys and acoustic navigation. IEEE Ocean'95, 1631-1642.

Tarantola, A., Valette, B., 1982. Generalized non-linear problems solved using the least squares criterion. Rev. Geophys. 20, 219-232. 